\section{Nora Hilda Hernandez Garcilazo, MD \\ Department of Internal Medicine, \\ Michigan State University, East Lansing, MI \\ Tushar J. Vachharajani, MD \\ Department of Nephrology and Hypertension, \\ Glickman Urological and Kidney Institute, Cleveland \\ Clinic, Cleveland, $\mathrm{OH}$; Professor, Cleveland Clinic \\ Lerner College of Medicine of Case Western Reserve \\ University, Cleveland, $\mathrm{OH}$}

\section{Mohamed Hassanein, MD}

Department of Nephrology and Hypertension,

Glickman Urological and Kidney Institute,

Cleveland Clinic, Cleveland, $\mathrm{OH}$

\section{Evamaria Anvari, MD}

Department of Nephrology and Hypertension,

Glickman Urological and Kidney Institute, Cleveland

Clinic, Cleveland, OH; Clinical Assistant Professor,

Cleveland Clinic Lerner College of Medicine of Case

Western Reserve University, Cleveland, $\mathrm{OH}$

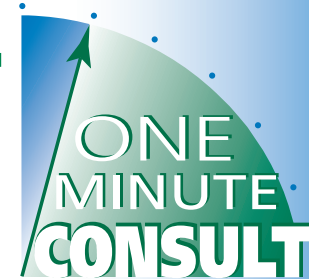

BRIEF ANSWERS

TO SPECIFIC

CLINICAL

QUESTIONS

\title{
: Can I place a peripherally inserted central catheter in my patient with chronic kidney disease?
}

A 45-year-old man is admitted to the hospital for sepsis secondary to osteomyelitis. He has diabetes mellitus, hypertension, and stage 3 chronic kidney disease (CKD), with a glomerular filtration rate of $46 \mathrm{~mL} / \mathrm{min} / 1.73 \mathrm{~m}^{2}$. He is treated with intravenous (IV) antibiotics and improves clinically. He will need 6 weeks of IV antibiotics after discharge. Should a peripherally inserted central catheter (PICC) be placed for IV access?

The decision to place a PICC must be
individualized for the patient. Current
guidelines do not provide explicit contraindications for creating permanent vascular access, but the general consensus is that poor candidates include those with advanced dementia, left ventricular ejection fraction less than $20 \%$, poor vasculature on imaging, or terminal illness (life expectancy $<6-12$ months). ${ }^{1}$ In addition, national guidelines and the American Board of Internal Medicine's Choosing Wisely initiative recommend against PICC placement in patients expected to need permanent dialysis access in the future (CKD stages 3-5). ${ }^{2}$

\section{PICC PROS: CONVENIENCE, LOW COST}

PICCs have become increasingly popular in recent years due to their ease of placement, convenience for patients, and cost-effective maintenance. Up to $56 \%$ of PICCs are placed to administer IV antibiotics. ${ }^{3}$

\section{PICC CONS: BLOOD VESSEL RISKS}

PICCs are highly associated with phlebitis, thromboembolism, central vein thrombosis, and stenosis of the involved vessels, which may

doi:10.3949/ccjm.88a.20173 obliterate the involved veins and prevent their use for future creation of a permanent dialysis access. ${ }^{4}$ Clinically diagnosed thrombosis has been reported to occur in $1 \%$ to $4 \%$ of patients with a PICC. However, in a 2000 study using venography to evaluate patency of the vessels, Allen et $\mathrm{al}^{5}$ reported a much higher incidence, with thrombosis evident in $23.3 \%$ of patients after PICC insertion.

Higher rates of thrombosis are associated with larger catheter sizes, the use of cephalic veins (due to smaller size compared with basilic veins), greater number of lumens, placement of multiple catheters, and patient factors including malignancy or history of venous thromboembolism. ${ }^{5,6}$ Central venous stenosis may also occur, although it is not as common as thrombosis. ${ }^{?}$

PICC insertion is also a strong independent risk factor for failure of an arteriovenous fistula, the preferred method of vascular access for hemodialysis. ${ }^{7} \mathrm{McGill}$ et al, ${ }^{8}$ in a 2016 observational study, found that PICC insertion before or after initiation of hemodialysis was associated with failure to transition to a form of permanent access, with only $24.7 \%$ of patients transitioning to a working arteriovenous fistula, and $11.5 \%$ transitioning to a functioning arteriovenous graft. This is very important because the transition from central venous access to an arteriovenous fistula or graft is associated with better survival and fewer hospitalizations, due to lower risk of serious infections such as endocarditis and bacteremia. ${ }^{8}$

In patients such as our 45-year-old man, an end-stage kidney disease (ESKD) life plan should be created with input from a nephrologist to determine early access needs and to avoid unnecessary procedures and complications, while also considering life expectancy
PICC insertion

is a strong independent risk factor for failure of an arteriovenous fistula 

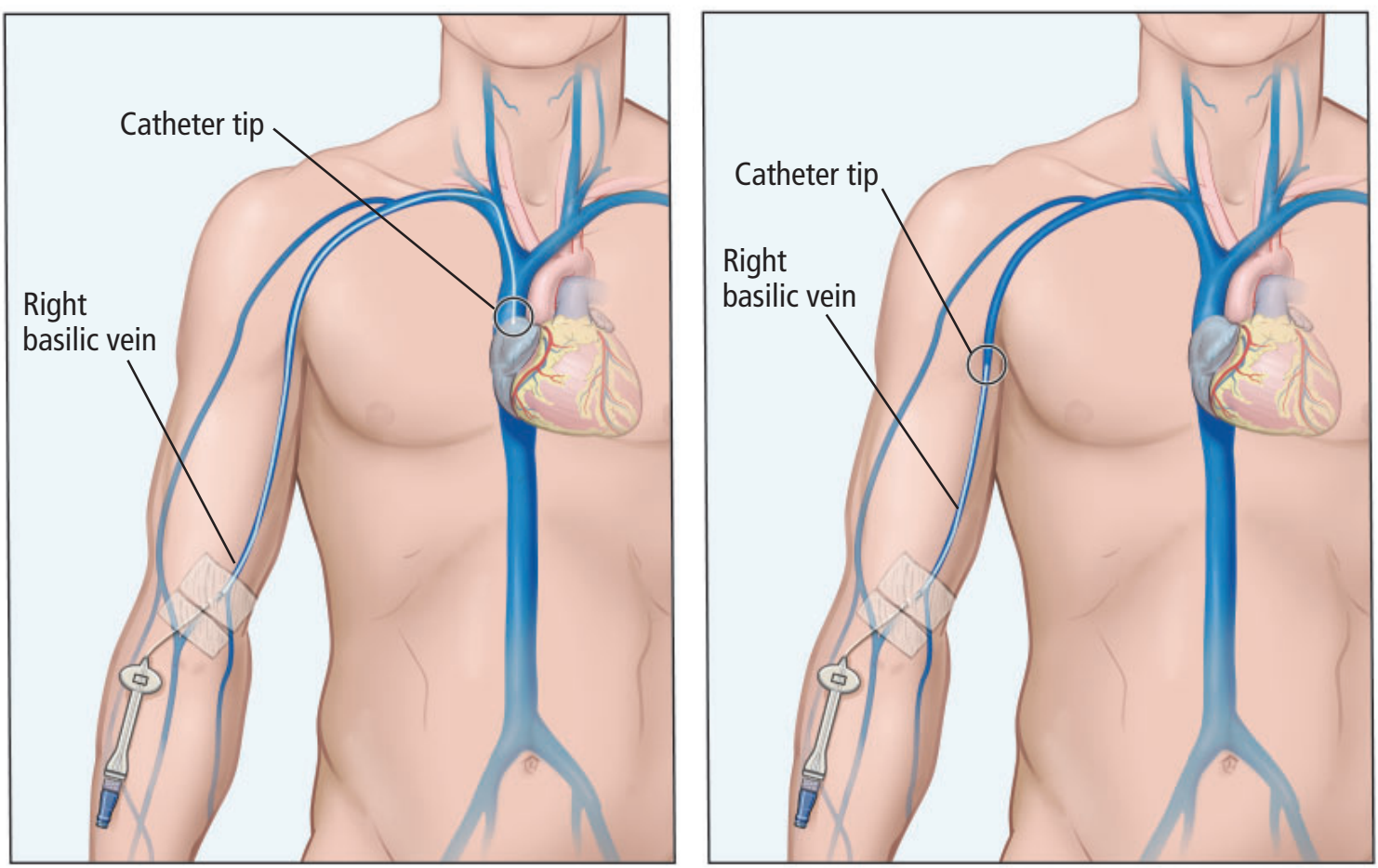

Figure 1. A peripherally inserted central catheter (left) is inserted at the right basilic vein, through the axillary and subclavian veins and into the superior vena cava. The midline catheter (right) is also inserted at the right basilic vein with the tip just below the axilla.

and kidney replacement alternatives. ${ }^{2}$

\section{CASE CONTINUED}

The team decides to place a PICC through the right basilic vein (Figure 1), and the infection resolves with 6 weeks of IV antibiotics.

However, 4 months later, he is readmitted for acute kidney injury and recurrence of osteomyelitis with a paravertebral abscess. The abscess is surgically drained, and the infectious disease consult recommends 8 weeks of IV antibiotics. After a thorough discussion with the nephrology team and the patient, the decision is made against placing a PICC.

\section{PICC ALTERNATIVES}

Unfortunately, all methods of IV access can produce venous damage, either by direct trauma at the puncture site or by device contact along the walls of the vein. ${ }^{9}$ It has been hypothesized that the more area within a vessel that a foreign object occupies, the greater the possibility of thrombosis due to increased stasis and direct-contact damage. ${ }^{3}$ However, midline catheters, which are also inserted into peripheral veins but occupy a smaller ending near the axilla, have also been associated with symptomatic venous thrombosis. ${ }^{4}$ Catheter location plays an important role, with guidelines suggesting avoiding cephalic, basilic, brachial, and subclavian veins. ${ }^{2,4}$

A proposed alternative to a PICC is a small-bore, 4-French or 6-French tunneled internal jugular catheter (Figure 2). It tends to last longer and is associated with fewer complications, decreasing the risk of central venous stenosis. ${ }^{10,11}$ A 2017 retrospective study by Bhutani et $\mathrm{al}^{10}$ found lower rates of deep vein thrombosis in tunneled small-bore central venous catheters than with PICCs, which may be explained by the shorter length of the catheter and better catheter-to-vein size ratio. However, whether they produce less damage to the peripheral vessels or cause central vein stenosis has not been fully studied. ${ }^{1}$

It has also been suggested that placement of internal jugular catheters by a skilled proceduralist with ultrasonographic and fluoroscopic guidance may result in less venous trauma, reducing the risk of vessel stenosis compared with nonguided methods. ${ }^{11}$ 


\section{HERNANDEZ GARCILAZO AND COLLEAGUES}

But even after an arteriovenous fistula has been successfully created, patients with ESKD requiring hemodialysis must continue vesselpreservation strategies as part of their ESKD management plan. ${ }^{1}$ If IV antibiotics are needed, it may be possible to select an agent that can be administered 3 times a week on dialysis days, using the functioning hemodialysis access. This will avoid the need for a different catheter, decreasing the risk of central venous stenosis and allowing for the creation of other arteriovenous fistulas if the current one fails. ${ }^{3}$

\section{Lifelong vessel-preservation strategies}

Patients who may progress to ESKD and may require hemodialysis access in the future should be identified early so that they can be provided with timely education regarding vessel preservation. This includes patients with stage 3 to stage $5 \mathrm{CKD}$, patients already on kidney replacement therapy such as hemodialysis or peritoneal dialysis, and patients who have a functional transplanted kidney. Such patients should be encouraged to advocate to preserve their vessels and work with the treatment team in balancing the risks and benefits of every intervention, including blood draws and use of IV and arterial devices. ${ }^{4,9}$ Medical alert bracelets and signs at the bedside of hospitalized patients with CKD indicating the need to restrict needle use is essential in educating and alerting the medical community. ${ }^{4}$

It has also been proposed that a nephrology consult be requested before placing a PICC in

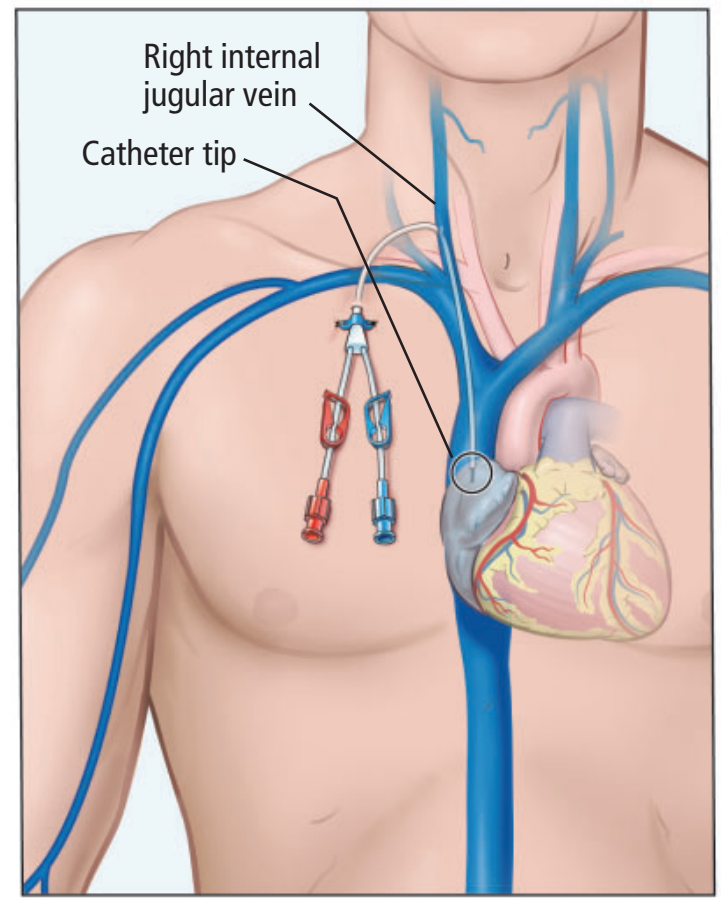

Figure 2. A small-bore (Hohn) catheter placed in the internal jugular vein is an alternative to a peripherally inserted central catheter.

patients with advanced CKD (stages 3-5). ${ }^{12} \mathrm{~Pa}$ tients and health professionals are encouraged to visit the website www.saveyourvein.org to further educate themselves on the importance of vein preservation. ${ }^{2}$

\section{DISCLOSURES}

The authors report no relevant financial relationships which, in the context of their contributions, could be perceived as a potential conflict of interest.

\section{REFERENCES}

1. Dumaine C, Kiaii M, Miller L, et al. Vascular access practice patterns in Canada: a national survey. Can J Kidney Health Dis 2018; 5:2054358118759675. doi:10.1177/2054358118759675

2. Lok CE, Huber TS, Lee T, et al. KDOQI clinical practice guideline for vascular access: 2019 update. Am J Kidney Dis 2020; 75(4 suppl 2):S1-S164. doi:10.1053/j.ajkd.2019.12.001

3. Shingarev R, Allon M. Peripherally inserted central catheters and other intravascular devices: how safe are they for hemodialysis patients? Am J Kidney Dis 2012; 60(4):510-513. doi:10.1053/j.ajkd.2012.07.003

4. Vachharajani TJ, Hassanein M, Liaqat A, Haddad N. Vessel preservation in chronic kidney disease. Adv Chronic Kidney Dis 2020; 27(3):177-182. doi:10.1053/j.ackd.2020.03.006

5. Allen AW, Megargell JL, Brown DB, et al. Venous thrombosis associated with the placement of peripherally inserted central catheters. J Vasc Interv Radiol 2000; 11(10):1309-1314. doi:10.1016/s1051-0443(07)61307-4

6. Chopra V, Flanders SA, Saint S. The problem with peripherally inserted central catheters. JAMA 2012; 308(15):1527-1528. doi:10.1001/jama.2012.12704

7. El Ters M, Schears GJ, Taler SJ, et al. Association between prior peripherally inserted central catheters and lack of functioning arteriovenous fistulas: a casecontrol study in hemodialysis patients. Am J Kidney Dis 2012; 60(4):601-608. doi:10.1053/j.ajkd.2012.05.007

8. McGill RL, Ruthazer R, Meyer KB, Miskulin DC, Weiner DE. Peripherally inserted central catheters and hemodialysis outcomes. Clin J Am Soc Nephrol 2016; 11(8):1434-1440. doi:10.2215/CJN.01980216

9. Saad T. Vein preservation and alternative venous access. Endovasc Today 2008; 32-36. https://evtoday.com/articles/2008-june/EVT0608_03-php. Accessed July 14, 2021.

10. Bhutani G, El Ters M, Kremers WK, et al. Evaluating safety of tunneled small bore central venous catheters in chronic kidney disease population: a quality improvement initiative. Hemodial Int 2017; 21(2):284-293. doi:10.1111/hdi.12484

11. Sasadeusz KJ, Trerotola SO, Shah H, et al. Tunneled jugular small-bore central catheters as an alternative to peripherally inserted central catheters for intermediate-term venous access in patients with hemodialysis and chronic renal insufficiency. Radiology 1999; 213(1):303-306. doi:10.1148/radiology.213.1.r99se12303

12. Williams AW, Dwyer AC, Eddy AA, et al. Critical and honest conversations: the evidence behind the 'Choosing Wisely' campaign recommendations by the American Society of Nephrology. Clin J Am Soc Nephrol 2012; 7(10):16641672. doi:10.2215/CJN.04970512

Address: Nora Hilda Hernandez Garcilazo, MD, Department of Internal Medicine, Michigan State University, Medical Arts Building, 1322 E. Michigan Ave. Suite 300, Lansing, MI 48912; herna644@msu.edu 